\title{
Linking Numerical and Analytical Models of Carbon Leakage
}

\author{
By Jared C. Carbone*
}

Carbon leakage describes the economywide response in carbon emission levels caused by abatement in one part of the economy. Most quantitative studies of carbon leakage predict that $10-30 \%$ of unilateral abatement efforts will be offset by emission increases (a positive net leakage effect) elsewhere in the world economy. This is the subject of intense policy focus in international climate policy debates because countries contemplating new policies to reduce domestic emissions worry that their efforts will be rendered costly and ineffective.

In a recent theoretical study, Fullerton, Karney and Baylis (2011) (henceforth FKB) identify a new potential source of carbon leakage effects which they refer to the Abatement Resource Effect (henceforth ARE). The intuition for this effect is that when stricter carbon regulation comes into effect in one part of the economy, it may draw basic factors of production away from other, carbon-intensive activities. The authors show that if this effect is strong enough, an economy may exhibit negative net leakage in response to the policy change. That is, emissions levels outside of the abating part of the economy may fall as these resources are drawn away and the scale of their operations shrink.

FKB identify the key parameters responsible for producing the net leakage effect in their model. Intuitively, it depends on a substitution parameter that governs the ease with which the economy can to shift out of the use of carbon services and into other resources within the regulated sector or region (a technique effect) and on a substitution parameter which governs the ease

\footnotetext{
* Department of Economics, University of Calgary, 2500 University Dr. N.W., Calgary, AB, T2N 1N4, Canada; Phone: 403-220-4094; Email: j.c.carbone@ucalgary.ca. Thanks to Don Fullerton for organizing the "Impacts of Unilateral Climate Change Policy" session at the 2013 AEA annual meeting and thanks to all of the session participants.
}

with which the economy can shift out of producing the goods derived from the regulated sector or region (a composition effect). It is the relationship between these two substitution patterns that determines the sign and magnitude of the leakage effect. If the composition effect dominates, the economy reduces the scale of the regulated sector, causing resources flow out to other, pollution-generating units of the economy and positive leakage occurs. If the technique effect dominates, then resources flow into the regulated sector as it shifts to a less carbon-intensive production technique. The scale and pollution levels of the other, resource-starved sectors fall, resulting in negative leakage.

While the intuition behind the result is clear, it is less clear from the differences in the structure of the FKB analytical model and the typical quantitative model from the carbon leakage literature in what settings the ARE is likely to exert an important influence on aggregate leakage results. There are three important differences. First, applied general equilibrium (or CGE) models - the primary tool used by researchers to quantify carbon leakage effects - contain deeply nested substitution patterns to capture key trade-offs between different fossil fuels and other, emission-intensive inputs such as electricity, that are primarily responsible for determining the character carbon leakage effects. In contrast, analytical models like the FKB model describe minimal structures designed to summarize these trade-offs in a simple way. Second, most CGE leakage experiments look at international sources of leakage and assume that factors are not internationally traded, precluding an ARE. There are few experiments exploring the effects of regulations imposed on a subset of polluting sectors within a region, a policy-relevant setting in which factor mobility may be high and the ARE may 
make an important contribution to leakage. Third, the FKB model abstracts from the most important source of positive leakage effects identified in the literature to date - inelastic fossil fuel supply. Inelastic fuel supply implies that stricter unilateral carbon regulations lead to lower fuel prices. This stimulates fuel demand and emissions elsewhere in the world economy.

In this paper, I use a detailed, calibrated CGE model to isolate the quantitative effect of the FKB leakage channels in the context of new carbon regulations in the electricity sector of the model economy. I first produce a version of the model in which the economic environment which conforms the main assumptions underlying the FKB analysis. I then introduce inelastic fossilfuel supply in the model, allowing me to put the quantitative significance of the ARE and negative leakage in the context of a model that also contains the single most important source of positive leakage effects identified in the literature to date. Thus, the research questions addressed in the paper are: What is the quantitative significance of the leakage channels identified by FKB in a calibrated model that conforms to the key assumptions underlying their analysis? What is the quantitative significance of these channels in a more comprehensive model of carbon leakage?

\section{Model Description}

I begin by reviewing the key assumptions of the FKB model and then proceed to summarize the features of the CGE model used in my policy experiments.

In the FKB model, a single representative agent consumes two goods $(X, Y)$ which are produced in competitive industries by combining inputs of a clean factor $(K)$ and carbon pollution services $(C) . C$ and $K$ are perfectly mobile across the two sectors. $K$ is assumed to be in fixed aggregate supply in the economy. The supply of $C$ is assumed to be perfectly elastic. Production of $X(Y)$ is subject to a tax on carbon inputs $\tau_{X}\left(\tau_{Y}\right)$. The revenue from the taxes is returned lump sum to the representative agent in the model. The agent has prefer- ences defined over $X, Y$ and $C$. The agent chooses the level of $X$ and $Y$ it consumes and views $C$ as quasi-fixed. Preferences are homothetic and $C$ is weakly separable from other goods.

The FKB analysis examines the leakage effects associated with the marginal increase in $\tau_{X}$. One can interpret this policy change as the effect of increasing the pollution tax on one sector of a closed, national economy or one region in a multiple-region economy with international trade. In what follows, I take the one-region, sectoralregulation interpretation of the model.

The strength of the ARE (and the prospect of negative leakage) depends on the relationship between $\sigma_{X}$, the elasticity of substitution between the inputs of the clean factor and carbon services in the production of $X$, and $\sigma_{U}$, the elasticity of substitution between $X$ and $Y$ in final consumption.

To evaluate negative leakage effects, I extend a model developed by Böhringer, Carbone and Rutherford (2011). It is a useful platform for the experiments that are the subject of this study because the structure and calibration is typical of the CGE models used to study carbon leakage effects. The model's structure and calibration is based on the GTAP 7.1 database which includes detailed national accounts on production and consumption (inputoutput tables) together with $\mathrm{CO}_{2}$ emissions based on data from the International Energy Agency for up to 112 regions and 57 sectors (Narayanan and Walmsley 2008).

I aggregate the 57 sectors provided by GTAP to 14 sectors that reflect sectorspecific differences in energy and trade intensity. The energy goods identified are coal, crude oil, natural gas, refined oil products, and electricity which allows me to distinguish energy goods by $\mathrm{CO}_{2}$ intensity and to capture the potential for fossilfuel switching. The GTAP dataset also features energy-and-trade-intensive (nonenergy) commodities that are exposed to unilateral climate policies. Among these, I include: chemical products; mineral products; iron and steel; non-ferrous metals; machinery and equipment; plant-based 
fibers; air, land and water transports. All other GTAP sectors are aggregated into a single commodity.

The production structure of the model follows the structure of the GTAP dataset. Firms in competitive, constant-returns-toscale industries combine capital, labor and intermediate inputs from the set of produced goods described above. The single representative agent in the regional economy consumes the same set of produced goods.

Figure 1 depicts the production structure (based on nested constant elasticity of substitution functions) for the sectors and the representative agent's final consumption bundle in the model. The elasticity of substitution for each nest is indicated at the top of each nest in the diagram. $Y_{g}$ indicates the level of output for activity $g$, where $g$ indexes the set of all sectors plus final consumption. In the top-level nest, a bundle of intermediate inputs (Materials) trades off against a bundle capital, labor and energy goods at a substitution elasticity of $\sigma_{K L E M, g}$. The individual commodities in the materials bundle substitute at rate $\sigma_{M, g}$. A bundle of capital and labor trades off against a bundle of energy goods at rate $\sigma_{K L E, g}$. Within the value-added bundle, capital and labor trade off at rate $\sigma_{K L, g}$. Within the energy bundle, electricity trades off against a bundle of fossil energy goods at rate $\sigma_{E}$. The substitution elasticity between the fossil energy goods in this bundle is $\sigma_{F E}$. No capital or labor is used in the final consumption bundle, thus the energy bundle (electricity and fossil energy) enters the top-level nest directly in the production of the final consumption good. Firms in fossil fuel resource sectors (coal, crude oil and natural gas) also require the use of natural resources which are in fixed supply and specific to each fuel sector. I calibrate the substitution elasticity between these inputs and a bundle of all other inputs (based on the same structure described in figure 1) in these sectors to imply specific values for the fossil fuel supply elasticities in the model. In the benchmark model, $\sigma_{K L E M, g}, \sigma_{M, g}, \sigma_{K L E, g}$ and $\sigma_{K L, g}$ are based on sector-specific, econometric esti- mates (Okagawa and Ban 2008). $\sigma_{E}$ and $\sigma_{F E}$ take on values of 0.5 and 0.75 across all sectors of the economy.

I aggregate the regional detail included in the GTAP data to the level of a single world region. In this case, the model corresponds to a closed economy with benchmark technologies based on the average input intensities in production and consumption across all world regions in the dataset. Combined with the assumptions that basic factors (capital and labor) are sectorally mobile and fossil fuel supplies are perfectly elastic, this model conforms to the assumptions in the single-region, multi-sector interpretation of the FKB model, where $X$ represents one domestic production sector. In the experiments presented here, $X$ represents the electricity sector.

\section{Policy Experiments}

I model the effect of moving from a benchmark equilibrium with no carbon regulation to a counterfactual equilibrium in which the electricity sector is subject to a carbon tax that is sufficiently high to produce a $20 \%$ reduction in emissions generated within the sector (and other sectors remain unregulated).

In the FKB model, the ARE (and the prospect of negative leakage) depends on the relative ease with which the economy can shift its composition away from the regulated sector and shift the technique of production within that sector to become less carbon intensive. It is the elasticity of substitution parameters, $\sigma_{X}$ and $\sigma_{U}$, that are responsible for controlling these trade offs in the FKB model. Electricity is the regulated sector in the application. Thus parameters in the CGE model that control the ease with which fossil fuels (the source of pollution) and the basic factor inputs can be substituted for one another within this sector will determine the strength of the technique effect (analogous to the role of $\sigma_{X}$ in FKB). Specifically, higher values of $\sigma_{K L E}$ in the sector will generate a stronger technique effect as it becomes easier for firms in the electricity industry to shift out of fossil fuel use and into capital and labor. The 


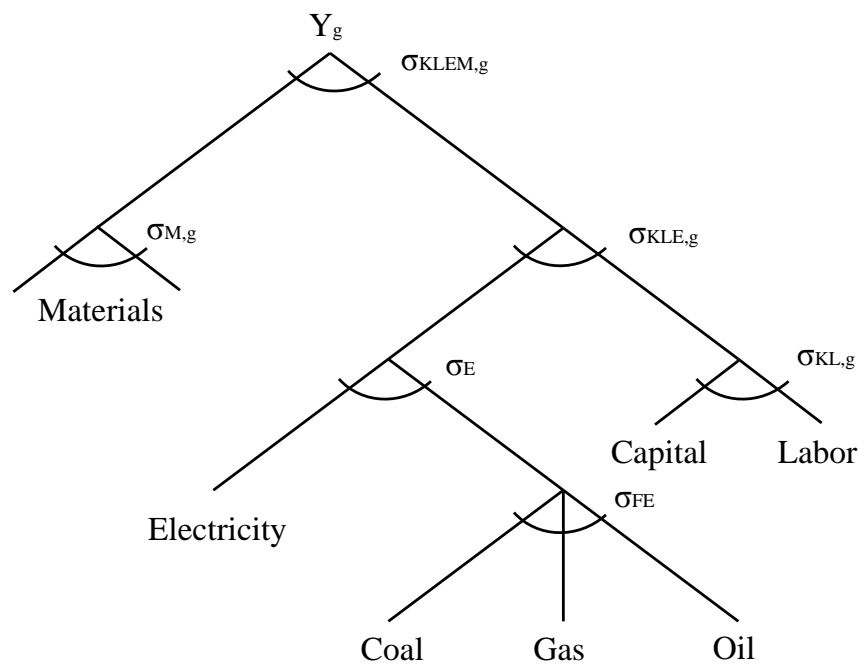

Figure 1. Nested Production Structure

value of $\sigma_{F E}$, the elasticity of substitution between coal, gas and oil within the fossil energy bundle in the sector may also have an important effect. These three fuels differ in the carbon emissions generated per unit of energy services delivered. Thus, the electricity industry will switch to lower carbon fuels (primarily natural gas) in response to carbon regulation. On one hand, lower values of $\sigma_{F E}$ should produce less fuel switching and more reliance on capital and labor inputs to generate output, yielding lower leakage rates. On the other hand, lower scope for fuel switching raises the overall cost of compliance with the carbon regulation, which could lead to a larger reduction in the scale of the electricity industry and lead to higher leakage rates.

The parameters in the CGE model that affect the degree to which the economy can substitute away from electricity inputs in unregulated sectors and in final consumption will determine the strength of the composition effect (analogous to the role of $\sigma_{U}$ in FKB). Higher values of $\sigma_{E}$ in these sectors will make replacing electricity inputs with other energy inputs easier, leading to higher leakage rates as this substitution pattern promotes lower output and pollution levels in the electricity sector as well as increase fuel use and pollution in other sectors. Lower values of $\sigma_{K L E}$ in these sectors may produce higher or lower leakage rates. It becomes less possible for firms to substitute away from using energy at lower values of this elasticity parameter. If the composition of energy use changes little, then electricity use remains relatively high after regulation, resulting in less leakage. If the composition of energy use shifts substantially away from electricity and toward fossil fuels then leakage rates may rise.

In the sensitivity analysis that I conduct, I vary the values of these three elasticity parameters $\left(\sigma_{K L E}, \sigma_{E}\right.$, and $\left.\sigma_{F E}\right)$ in either the electricity sector or the non-electicity sectors and the final consumption bundle. I halve or double the benchmark values of these parameters in the model and run all combinations of these value across the three parameters. I first change the values in the electricity sector alone, holding the values for non-electricity sectors at benchmark levels. I then change the values in all nonelectricity sectors and final consumption in tandem and hold electricity sector values at benchmark levels.

The last dimension of sensitivity analysis varies the supply elasticities of fossil fuels in the model. In the Böhringer, Carbone 
and Rutherford (2011) model, these elasticities are set equal to $0.25,0.5$ and 1 for natural gas, oil and coal respectively. To reproduce the conditions in the FKB model, I assume fuel supplies are all perfectly elastic. I also perform runs of the model with the Böhringer, Carbone and Rutherford (2011) benchmark values as well as runs in which fuel supplies are perfectly inelastic. The past experiments on carbon leakage typically identify the fuel-supply channel as the main driver of leakage effects, whereby the lower world prices of these fuels caused by lower demand in regulated regions causes uptake in unregulated parts of the economy. This effect is stronger when fuel supply is less elastic. Therefore, models with less elastic fuel supply are less likely to generate net negative leakage effects.

The discussion of the results of the leakage experiments proceeds in the following way. I first describe the results for the experiments run at the benchmark elasticity values used in Böhringer, Carbone and Rutherford (2011). Thus, all of the key substitution and fuel-supply elasticities are set at the benchmark values from that study. These results are intended to give a central estimate for the sign and magnitude of carbon leakage effect - a prediction of the model at a set of parameter values representative of the assumptions used in previous studies of carbon leakage effects. I follow this with an examination of how leakage rates change when I vary the key elasticity assumptions in the model.

Overall, approximately $9.5 \%$ of the reduction in electricity sector emissions is offset by positive carbon leakage in the rest of the economy in the central-case counterfactual experiment. By sector (i.e. how much emission changes from a given sector contribute to the aggregate leakage rate), most of the positive emission responses come from the chemicals industry, increases in final consumption, non-metallic minerals production and increases in the sector that is an aggregate of non-energy-intensive sectors in the economy. By fuel (i.e. how much the combustion of a given fuel contributes to the aggregate leakage rate), all fuels exhibit increased use. The positive leakage stems primarily from the use of coal, followed by natural gas and oil. In the central case simulations, fuel supply is inelastic so the imposition of carbon regulation causes the price of fuels demanded by that sector to fall, inducing increased demand for these fuels elsewhere in the economy. Coal is the primary fuel used in the electricity industry, followed by natural gas. Thus, these fuels are subject to the largest leakage effects in these experiments. Coal is used in a number of heavy industries. Natural gas is also used in these sectors as well as for heating and cooling in final consumption and manufacturing, for example. Thus there is uptake in the use of these fuels in these sectors.

Table 1 depicts the results of the sensitivity analysis with respect to the key elasticity parameters. The left half of the table describes the results of the experiments which vary the electricity-sector parameters. The rows of the table enumerate the different assumptions regarding the values of the substitution elasticities between fossil fuels $\left(\sigma_{F E}\right)$ and between the value-added bundle and the energy bundle $\left(\sigma_{K L E}\right)$. An entry of "High" in the table indicates a doubling of the benchmark value of a given elasticity, an entry of "Low" indicates a halving and "Ref" reproduces the benchmark value. The columns of the table enumerate the different fossil fuel supply elasticity assumptions. An entry of "High" corresponds to perfectly elastic supply, an entry of "Low" to perfectly inelastic supply and "Ref" reproduces the benchmark values from the Böhringer, Carbone and Rutherford (2011) model.

When fuel supply is perfectly elastic, leakage rates are small and positive (0-5\%). Increasing the possibilities for fuel substitution (higher $\sigma_{F E}$ ) leads to lower leakage rates as it becomes less costly for firms in the electricity industry to comply with the new carbon regulation. Increasing $\sigma_{K L E}$ leads to lower leakage when fuel supply is perfectly elastic and higher leakage when it is not. When fuel supply is elastic, a higher $\sigma_{K L E}$ value leads to more substitution away from energy into capital and labor, leading to the ARE effect. When fuel supply is not perfectly elastic, more substitution 
Table 1-Aggregate Leakage Rates by Elasticity Assumption

\begin{tabular}{|c|c|c|c|c|c|c|c|c|c|}
\hline \multicolumn{5}{|c|}{ Electricity Sector } & \multicolumn{5}{|c|}{ Non-Electricity Sectors } \\
\hline \multirow[t]{2}{*}{$\sigma_{F E}$} & \multirow[t]{2}{*}{$\sigma_{K L E}$} & \multicolumn{3}{|c|}{$\begin{array}{l}\text { Fuel Supply } \\
\text { Elasticities }\end{array}$} & \multirow[t]{2}{*}{$\sigma_{E}$} & \multirow[t]{2}{*}{$\sigma_{K L E}$} & \multicolumn{3}{|c|}{$\begin{array}{l}\text { Fuel Supply } \\
\text { ElASTICITIES }\end{array}$} \\
\hline & & Low & Ref & High & & & Low & Ref & High \\
\hline \multirow{3}{*}{ Low } & High & 27.29 & 13.61 & 1.46 & \multirow{3}{*}{ High } & Low & 24.74 & 19.12 & 15.00 \\
\hline & Ref & 24.66 & 13.60 & 3.53 & & Ref & 23.85 & 17.14 & 12.17 \\
\hline & Low & 22.68 & 13.70 & 5.34 & & High & 22.95 & 14.25 & 7.18 \\
\hline \multirow{3}{*}{ Ref } & High & 20.67 & 10.33 & 0.90 & \multirow{3}{*}{ Ref } & Low & 18.37 & 11.33 & 4.85 \\
\hline & Ref & 17.43 & 9.45 & 2.09 & & Ref & 17.43 & 9.45 & 2.09 \\
\hline & Low & 15.07 & 8.81 & 2.97 & & High & 16.59 & 6.76 & -2.70 \\
\hline \multirow{3}{*}{ High } & High & 15.62 & 7.54 & 0.35 & \multirow{3}{*}{ Low } & Low & 13.40 & 5.98 & -1.26 \\
\hline & Ref & 12.79 & 6.55 & 0.91 & & Ref & 12.54 & 4.20 & -3.92 \\
\hline & Low & 10.89 & 5.90 & 1.25 & & High & 11.94 & 1.71 & -8.50 \\
\hline
\end{tabular}

Note: Leakage rates displayed in the table are defined as $\left(E_{1}^{n e}-E_{0}^{n e}\right) /\left(E_{0}^{e}-E_{1}^{e}\right)$ where $E_{1,0}^{n e}$ are the emissions levels summed across all non-electricity sectors post and pre policy respectively and $E_{0,1}^{e}$ are the emissions levels in the electricity sector pre and post policy respectively.

away from energy leads to a larger reduction in the price of fuels, causing a stronger, positive fuel-supply leakage effect. There is no scenario that generates net negative leakage.

The right half of table 1 depicts the results of the sensitivity analysis with respect to the elasticity parameters in the nonelectricity sectors. The display of the results follows that of the electricity-sector analysis with the exception that $\sigma_{E}$, the elasticity parameter that controls substitution between electricity inputs and the bundle of fossil energy inputs, replaces $\sigma_{F E}$ in the first column.

Lowering the value of $\sigma_{E}$ always lowers leakage rates, as consumers and firms find it more difficult to substitute away from electricity. Raising the value of $\sigma_{K L E}$ always lowers leakage rates because firms may more easily substitute away from the bundle of energy goods. These experiments do yield cases with negative net leakage when fuel supplies are elastic, when substitution away from energy inputs is more of a possibility, and when electricity cannot be easily replaced in the bundle of energy goods.

In summary, I find net negative leakage in the configurations of the model that best match the assumptions in the FKB analy- sis. Thus the FKB analytical model and the numerical model in this study have a correspondence. Nevertheless, positive net leakage effects dominate the results. The most important determinant of this result is the inclusion of inelastic fuel supply in the model. However, I find that the benchmark assumptions used in the literature regarding the scope for substitution between energy goods and basic factors contribute to this result as well.

\section{REFERENCES}

Böhringer, Christoph, Jared C. Carbone, and Thomas F. Rutherford. 2011. "Embodied Carbon Tariffs." National Bureau of Economic Research, Working Paper No. 17376.

Fullerton, Don, Daniel Karney, and Kathy Baylis. 2011. "Negative Leakage." National Bureau of Economic Research, Working Paper No. 17001.

Narayanan, Badri, and Terrie L. Walmsley. 2008. "The GTAP 7 Data Base Documentation." Purdue University, Center for Global Trade Analysis.

Okagawa, Azusa, and Kanemi Ban. 2008. "Estimation of Substitution Elasticities for CGE Models." Osaka University mimeo. 